\title{
Canonical quantization of cylindrically symmetric models
}

\author{
Guillermo A. Mena Marugán \\ Instituto de Matemáticas y Física Fundamental, C.S.I.C., Serrano 121, 28006 Madrid, Spain
}

(Received 18 October 1995)

\begin{abstract}
We carry out the canonical quantization of the Levi-Civita family of static and cylindrical solutions. The reduced phase space of this family of metrics is proved to coincide with that corresponding to the Kasner model, including the associated symplectic structures, except that the respective domains of definition of one of the phase space variables are not identical. Using this result, we are able to construct a quantum model that describes spacetimes of both the Levi-Cività and the Kasner type, and in which the three-dimensional spatial topology is not uniquely fixed. Finally, we quantize to completion the subfamily of Levi-Cività solutions which represent the exterior gravitational field of a straight cosmic string. These solutions are conical geometries, i.e., Minkowski spacetime minus a wedge. The quantum theory obtained provides us with predictions about the angular size of this wedge.
\end{abstract}

PACS number(s): $04.60 . \mathrm{Kz}, 98.80 . \mathrm{Hw}$

\section{INTRODUCTION}

Spacetimes with cylindrical symmetry are employed in general relativity to describe a variety of physically interesting situations. The general form of the vacuum fourdimensional metric for cylindrically symmetric spacetimes was first obtained by Kompaneets [1]. Particular examples of such a metric provide exact solutions to Einstein's equations which represent cylindrical gravitational waves [2-4]. These waves can be superimposed on the exterior gravitational field created by a massive static cylinder [2]. Furthermore, the existence of cylindrical symmetry allows one to define a locally measurable gravitational energy, which can be proved to be localized for propagating cylindrical waves [3].

Cylindrical symmetry, on the other hand, guarantees that the spacetime possesses two spacelike Killing vectors [5]. Thanks to this fact, once a cylindrical solution is known, one can apply to it the generalized soliton transformation of Belinskii and Zakharov [6] to generate new vacuum solutions. The cylindrical metrics which have been more frequently used as seeds for the Belinskii-Zakharov technique are the Levi-Cività (LC) family of solutions [7,8]. In this way, it has been possible to construct a series of cylindrical solutions which describe the propagation of gravitational waves on a LC background [5].

The LC metrics are the most general static and cylindrically symmetric solution to Einstein's equations in a vacuum, and can be expressed in the form

$$
d s^{2}=C \rho^{2 p^{2}-\frac{1}{2}}\left(-d t^{2}+d \rho^{2}\right)+A B \rho^{1+2 p} d \phi^{2}+\frac{A}{B} \rho^{1-2 p} d z^{2},
$$

where $A, B$, and $C$ are three positive constants and $p$ a real constant. The time coordinate $t$ and the axial coordinate $z$ take on real values, $\rho \in \mathbb{R}^{+}$is the radial coordinate, and $\phi$ $\in S^{1}$ the polar angle.

Except when $p=\frac{1}{2}$, metrics (1.1) can be interpreted as the exterior gravitational field produced by a static cylinder $[2,7]$. For cylinders made of ordinary matter, i.e., with positive mass, we have $p>\frac{1}{2}$ [2]. When $p<\frac{1}{2}$, the mass of the cylinder must be negative, and the gravitational field described by metric (1.1) can be regarded as repulsive [4]. All metrics (1.1) with $p \neq \frac{1}{2}$ present a naked singularity at $\rho=0$. This singularity can be reinterpreted as a line source, which can be represented by an appropriate stress-energymomentum tensor on the axis $\rho=0$ [9].

When $p=\frac{1}{2}$, a suitable rescaling of the coordinates $t, \rho$, and $z$, which leaves invariant their respective domains of definition, leads to the subfamily of metrics

$$
d s^{2}=-d t^{2}+d \rho^{2}+\frac{A B}{C} \rho^{2} d \phi^{2}+d z^{2} .
$$

For $C=A B$ this is the metric of Minkowski spacetime in cylindrical coordinates. Of much more physical interest, nevertheless, is the case $C>A B$. Metric (1.2) describes then a conical spacetime, i.e., flat spacetime minus a wedge [10]. The angular size of this wedge is

$$
\Delta=2 \pi\left(1-\sqrt{\frac{A B}{C}}\right) .
$$

Such a conical geometry can actually be interpreted as the exterior field of a uniform, static, and infinite straight cosmic string with linear energy density equal to $2 \Delta[10,11]$ (we use units where $16 \pi G=c=\hbar=1$ ). This kind of topological defect [12] could play a relevant role in cosmology [10], as it could serve as seeds for eternal inflation [13], and provide the density fluctuations necessary for galaxy formation [14]. Cosmics strings would also have observational gravitational effects if they exist. They should produce a steplike discontinuity in the microwave background [15], and act as gravitational lenses, forming double images of astrophysical objects [16]. In the case of a straight cosmic string, for instance, the angular separation between these double images would be proportional to the deficit angle $\Delta$ of the exterior conical geometry [10].

In this work, we will construct a quantum theory for the description of the cylindrically symmetric LC spacetimes, and apply it to the study of different physical situations. In order to achieve this goal, we will follow the extension of 
Dirac's canonical quantization program [17] elaborated by Ashtekar [18]. This extended program has already proved extremely successful in dealing with the quantization of gravitational minisuperspace models [19-21]. In the case of the LC spacetimes, the quantization procedure to be employed will consist of the following steps. We will first find the symplectic structure of the associated reduced phase space. In this space, we will choose a complete set of elementary observables which form a Lie algebra under the Poisson brackets determined by the symplectic structure. These observables will be represented as operators acting on a vector space, in such a way that their commutators reproduce the corresponding classical Poisson brackets. We will then select the inner product in the representation constructed by imposing a set of reality conditions $[18,22]$, namely, by promoting the complex conjugation relations between classical elementary observables to adjointness requirements on quantum operators.

This paper is organized as follows. Section II is devoted to the canonical quantization of the LC metrics. In Sec. III we construct a quantum model that describes the LC and the Kasner [8] families of solutions altogether. The quantization of the subfamily of LC metrics that represent the exterior field of a straight cosmic string is presented in Sec. IV. We comment there on the type of predictions that can be extracted from the quantum theory obtained. Finally, we summarize and conclude in Sec. V.

\section{QUANTUM ANALYSIS OF THE LC SOLUTIONS}

The LC metrics (1.1) provide the most general static, cylindrical solution to Einstein's equations in vacuum. We can regard these metrics as the classical solutions of a cylindrically symmetric minisuperspace model in which all metric functions, and thus all degrees of freedom, depend exclusively on the radial coordinate $\rho$, and not on time. The Hamiltonian of such a minisuperspace model, obtained as a linear combination of all the first-class constraints of the system [17], must then dictate the evolution of the metric functions in the radial coordinate, rather than generating a true time evolution. In spite of this peculiarity, we will show that the quantization of the LC spacetimes poses no special difficulty if one interprets all gravitational variables as complex-valued functions on phase space, as proposed by Ashtekar [18].

In order to quantize the LC family of solutions, we will first prove that, with a reinterpretation of the spacetime coordinates, the LC and the Kasner metrics coincide except for the sign of the parameter $C$ appearing in Eq. (1.1). The Kasner solutions (i.e., the Bianchi type I diagonal metrics) can be written in the form [20]

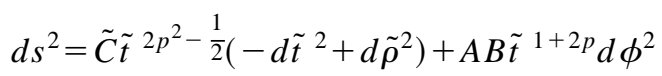

$$
\begin{aligned}
& +\frac{A}{B} \tilde{t}^{1-2 p} d z^{2},
\end{aligned}
$$

$\tilde{t} \in \mathbb{R}^{+}$being the time coordinate, $(\tilde{\rho}, \phi, z)$ a set of real spatial coordinates, $A, B$, and $\tilde{C}$ positive constants, and $p$, in principle, a real constant. Let us assume that, in the above equation, $\tilde{\rho}, z \in \mathbb{R}$ and $\phi \in S^{1}$. Identifying the coordinates $\rho$ and $t$ of Eq. (1.1) with $\tilde{t}$ and $\tilde{\rho}$, respectively (recall that $\rho, \tilde{t}$ $\in \mathbb{R}^{+}$and $\left.t, \tilde{\rho} \in \mathrm{R}\right)$, it is now obvious that the LC solutions adopt the same expression as the Kasner metrics (2.1), the only difference being that $\tilde{C}=-C<0$ in the LC case, whereas $\tilde{C}>0$ for the Kasner spacetimes.

As a consequence, the quantization of the LC solutions can be made by quantizing the Kasner metrics (2.1), provided that one replaces the nonholonomic constraint $\tilde{C}>0$ with $\tilde{C}<0$. In fact, the Kasner solutions have already been quantized [20,21]. Reference [20] contains a detailed analysis of the canonical quantization of these metrics which employs the same kind of notation and mathematical language used in this work. In particular, it was shown that the reduced phase space of the Kasner model has a symplectic structure given by

$$
\Gamma=d A \wedge d P_{A}+d p \wedge d P_{p}
$$

where

$$
P_{A}=p \ln B-\frac{1}{2} \ln \tilde{C}, \quad P_{p}=A(\ln B-2 p) .
$$

It was also argued that, as long as the spatial coordinates $\phi$ and $z$ are physically undistinguishable, one should restrict the parameter $p$ to be positive so that each possible fourgeometry described by a metric of the form (2.1) is taken into account only once. In our case, however, the symmetries and domains of definition of $\phi$ and $z$ are clearly different. So we must let $p$ run over the whole real axis if all possible geometries are to be considered.

We thus have that, for the Kasner metrics, $p \in \mathbb{R}$ and $A, B, \tilde{C} \in \mathbb{R}^{+}$. Then, from Eq. (2.3),

$$
P_{A}, P_{p} \in \mathbb{R} .
$$

The symplectic structure of the reduced phase space determined by Eq. (2.2) can therefore be interpreted as that corresponding to the cotangent bundle over $\mathbb{R}^{+} \times \mathbb{R}$.

For the LC metrics, on the other hand, $p, A$, and $B$ take on the same ranges of values as in the Kasner model, but now $\tilde{C} \in \mathrm{R}^{-}$. Hence, although we still get

$$
A \in \mathbb{R}^{+} \quad \text { and } \quad p, P_{p} \in \mathbb{R},
$$

the variable $P_{A}$ becomes complex:

$$
P_{A}=p \ln B-\frac{1}{2} \ln |\tilde{C}|-\frac{\pi}{2} i \in \mathbb{R}-\frac{\pi}{2} i,
$$

where we have taken $\ln (-1)=\pi i$. Notice that, the imaginary part of $P_{A}$ being constant, one can always absorb it by shifting the origin of $P_{A}$ in the complex plane. In this way, instead of working with the $P_{A}$ complex for the LC metrics, one can simplify calculations by introducing the new variable

$$
\tilde{P}_{A}=P_{A}+\frac{\pi}{2} i=p \ln B-\frac{1}{2} \ln |\tilde{C}|,
$$

which is real in the analyzed case: 


$$
\tilde{P}_{A} \in \mathbb{R} .
$$

The symplectic form (2.2) can now be rewritten

$$
\Gamma=d A \wedge d \tilde{P}_{A}+d p \wedge d P_{p} .
$$

From Eqs. (2.5) and (2.8), we then conclude that, for the LC spacetimes, the symplectic structure of the reduced phase space can be identified with that of the cotangent bundle over $\mathrm{R}^{+} \times \mathrm{R}$. This is precisely the symplectic structure obtained for the Kasner model. The only physical difference is that, in terms of the reduced phase space variables employed, the parameter $\tilde{C}$ of Eq. (2.1) adopts the following expressions for the Kasner and the LC solutions:

$$
\begin{gathered}
\tilde{C}=\exp \left(4 p^{2}+2 \frac{p P_{p}}{A}-2 P_{A}\right), \\
\tilde{C}=-\exp \left(4 p^{2}+2 \frac{p P_{p}}{A}-2 \tilde{P}_{A}\right),
\end{gathered}
$$

respectively.

A complete set of elementary variables in the reduced phase space of the LC metrics is formed by $p, P_{p}, A$, and the generalized momentum

$$
L_{A}=A \tilde{P}_{A} .
$$

Since these are reduced phase space variables, they all are gravitational observables. In addition, they form a Lie algebra under Poisson brackets, the only nonvanishing brackets being

$$
\left\{A, L_{A}\right\}=A, \quad\left\{p, P_{p}\right\}=1 .
$$

To quantize the system, we will represent these elementary observables as operators acting on the vector space of complex functions $\Psi(A, p)$ over $\mathbb{R}^{+} \times \mathbb{R}$, each function $\Psi(A, p)$ corresponding to a quantum state. The action of such operators will be given by

$$
\begin{gathered}
\hat{A} \Psi(A, p)=A \Psi(A, p), \quad \hat{L}_{A} \Psi(A, p)=-i A \partial_{A} \Psi(A, p), \\
\hat{p} \Psi(A, p)=p \Psi(A, p), \quad \hat{P}_{p} \Psi(A, p)=-i \partial_{p} \Psi(A, p) .
\end{gathered}
$$

These definitions guarantee that the introduced operators form the same algebra under commutators as their classical counterparts under Poisson brackets.

We can now fix the inner product in the representation constructed by promoting the reality conditions on the elementary observables to adjointness requirements on quantum operators [22]. Since the chosen elementary observables are real, we must demand that the operators $(2.13),(2.14)$ be self-adjoint. These Hermiticity conditions determine the unique inner product (up to a positive global factor)

$$
\langle\Phi, \Psi\rangle=\int_{\mathbb{R}^{+}} \frac{d A}{A} \int_{\mathbb{R}} d p \Phi^{*}(A, p) \Psi(A, p),
$$

where $\Phi^{*}$ is the complex conjugate to $\Phi$. The Hilbert space of physical states is thus $L^{2}\left(\mathbb{R}^{+} \times \mathbb{R}, d A d p / A\right)$. This completes the quantization of the LC solutions.

\section{QUANTUM MODEL WITH NONFIXED THREE-TOPOLOGY}

In this section, we will elaborate a quantum model which could describe both the LC and the Kasner families of solutions as a whole. Since the topology of the surfaces of constant time for these two types of classical spacetimes are different, we notice that the model to be quantized should not possess a uniquely fixed spatial topology.

We recall that both the Kasner and the LC solutions can be expressed in the form (2.1), with $A, B \in \mathbb{R}^{+}$and $p \in \mathbb{R}$. Metrics (2.1) correspond to Kasner or LC solutions, respectively, depending on whether $\tilde{C}$ is positive or negative. In both cases, the symplectic form on the reduced phase space is provided by formulas (2.2), (2.3). Suppose then that one performs a time reversal on the Kasner spacetimes. This time reversal can be seen to change the global sign of the symplectic form (2.2), although it does not alter the fourdimensional geometry. In fact, the same flip of sign can be obtained in Eq. (2.2) by means of the transformation $A \rightarrow-A$ and $B \rightarrow-B$, which leaves invariant metrics (2.1). Hence, instead of using Eq. (2.2), we can equivalently employ the following symplectic form for the Kasner model:

$$
\Gamma=-\left(d A \wedge d P_{A}+d p \wedge d P_{p}\right) .
$$

Let us define now

$$
\begin{aligned}
& \tilde{A}=\epsilon A, \quad \tilde{B}=\epsilon B, \\
& R=-\frac{\tilde{C}}{\tilde{A}}, \quad S=\frac{\tilde{A}}{\tilde{B}},
\end{aligned}
$$

where $\epsilon=1$ for the LC spacetimes, and $\epsilon=-1$ for the Kasner solutions. Then, metrics (2.1) adopt the expression

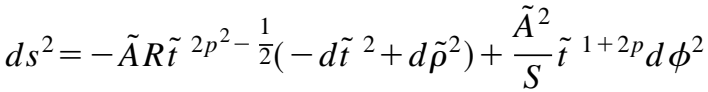

$$
\begin{aligned}
& +S \tilde{t}^{1-2 p} d z^{2}
\end{aligned}
$$

with

$$
p \in \mathbb{R}, \quad R, S \in \mathbb{R}^{+}
$$

and

$$
\tilde{A} \in \begin{cases}\mathbb{R}^{+} & \text {for } \mathrm{LC} \text { solutions, } \\ \mathbb{R}^{-} & \text {for Kasner solutions. }\end{cases}
$$

On the other hand, making use of relations (2.3) and (2.7), it is not difficult to check that the symplectic forms (3.1) and (2.9), valid on the respective reduced phase spaces of the Kasner and the LC models, can both be rewritten as

$$
\Gamma=d \tilde{A} \wedge d \Pi_{\tilde{A}}+d p \wedge d \Pi_{p},
$$

where the momenta $\Pi_{\tilde{A}}$ and $\Pi_{p}$ are 


$$
\Pi_{\tilde{A}}=-p \ln S-\frac{1}{2} \ln R, \quad \Pi_{p}=-\tilde{A}(\ln S+2 p-1) .
$$

Equations (3.5), (3.6), and (3.8) imply that, for the LC metrics,

$$
p, \Pi_{p}, \Pi_{\tilde{A}} \in \mathbb{R} \quad \text { and } \quad \tilde{A} \in \mathbb{R}^{+},
$$

whereas, in the Kasner case,

$$
p, \Pi_{p}, \Pi_{\tilde{A}} \in \mathbb{R} \quad \text { and } \tilde{A} \in \mathbb{R}^{-} .
$$

Thus, the reduced phase spaces of the two analyzed families of solutions differ only in the range of values allowed for the canonical variable $\tilde{A}$. This opens the possibility of simultaneously considering these two kinds of spacetimes by permitting that $\tilde{A}$ run over the whole real axis. In doing this, one is nevertheless forced to include as acceptable the value $\tilde{A}=0$, which, from expressions (3.4) and (3.8), can be reached only in the limit of degenerate metrics.

Actually, the symplectic structure (3.7) for the family of metrics (3.4) can be proved to coincide with that of reduced general relativity only as far as such metrics are not degenerate. This requirement of nondegeneracy guarantees that the parameters $\left(\tilde{A}, p, \Pi_{\tilde{A}}, \Pi_{p}\right)$ provide in fact a good set of coordinates in the reduced phase space of the system, because one can show then that these parameters are analytic functions of the metric of the model and of its first derivatives. However, if one regards the parameters $\left(\tilde{A}, p, \Pi_{\tilde{A}}, \Pi_{p}\right)$ as fundamental canonical variables on the reduced phase space, it should be clear that, from the point of view of the symplectic structure (3.7), the inclusion of the surface $\tilde{A}=0$ does not bring on any singularity. What one gets in this way is just an extension of reduced general relativity for the model (3.4) which reproduces exactly Einstein's theory when the metric is nondegenerate, i.e., when $\tilde{A}$ takes on either positive or negative values, corresponding to LC or Kasner spacetimes, respectively.

Admitting then that $\tilde{A} \in \mathbb{R}$, the symplectic structure determined by (3.7) can be identified with that of the cotangent bundle over $\mathbb{R} \times \mathbb{R}$. In order to quantize the system, we can choose the canonical variables $\tilde{A}, \Pi_{\tilde{A}}, p$, and $\Pi_{p}$ as a complete set of real observables, and represent them as the following operators acting on the vector space of complex functions $\Psi(\tilde{A}, p)$ over $\mathrm{R} \times \mathrm{R}$ :

$$
\begin{array}{ll}
\hat{\tilde{A}} \Psi(\tilde{A}, p)=\tilde{A} \Psi(\tilde{A}, p), & \hat{\Pi}_{\tilde{A}} \Psi(\tilde{A}, p)=-i \partial_{\tilde{A}} \Psi(\tilde{A}, p), \\
\hat{p} \Psi(\tilde{A}, p)=p \Psi(\tilde{A}, p), & \hat{\Pi}_{p} \Psi(\tilde{A}, p)=-i \partial_{p} \Psi(\tilde{A}, p),
\end{array}
$$

whose only nonvanishing commutators are

$$
\frac{1}{i}\left[\hat{\tilde{A}}, \hat{\Pi}_{\tilde{A}}\right]=1, \quad \frac{1}{i}\left[\hat{p}, \hat{\Pi}_{p}\right]=1 .
$$

The elementary operators (3.11), (3.12) must be self-adjoint, because they represent real classical observables. These reality conditions select the physical inner product

$$
\langle\Phi, \Psi\rangle=\int_{\mathrm{R} \times \mathrm{R}} d \tilde{A} d p \Phi^{*}(\tilde{A}, p) \Psi(\tilde{A}, p) .
$$

The Hilbert space of physical states is thus $L^{2}(\mathbb{R} \times \mathbb{R}, d \tilde{A} d p)$.

It is worth remarking that, according to our discussion above [see Eq. (3.6)], we can interpret the sign of the expectation value of $\tilde{A}$ in any quantum state $\Psi$,

$$
\eta=\operatorname{sgn}\left(\frac{\langle\Psi, \hat{\tilde{A}} \Psi\rangle}{\langle\Psi, \Psi\rangle}\right),
$$

as the prediction of a four-dimensional geometry being of either the LC $(\eta=1)$ or the Kasner type $(\eta=-1)$. Let us suppose then that, e.g., $\eta=-1$ for a particular state $\Psi_{0}$, and that the projection of $\Psi_{0}$ on the sector $\tilde{A}>0$,

$$
\Psi_{0}^{+}(\tilde{A}, p)=\int_{\mathrm{R}^{+}} d A \delta(\tilde{A}-A) \Psi_{0}(A, p)
$$

is normalizable with respect to the inner product (3.14). At least formally, one can understand projection (3.16) as the collapse of the wave function $\Psi_{0}$ produced by a measurement in which one just detected whether the geometry is static and cylindrically symmetric (i.e., of the LC type). After such an irreversible collapse, it is obvious that the expectation value of $\tilde{A}$ becomes positive, so that, from Eq. (3.15), we will have that $\eta=1$ in the analyzed quantum state. In this sense, the collapse of the wave function $\Psi_{0}$ to $\Psi_{0}^{+}$, implied by the physical measurement explained above, can be interpreted in our quantum theory as leading to a change in the spatial topology, for the LC and the Kasner surfaces of constant time possess, respectively, different topologies.

Some comments are in order at this point. We first note that no change of spatial topology can be achieved classically in our model, since the variable $\tilde{A}$ is a constant of motion for all metrics of the form (3.4). In addition, it is worth insisting that the change of topology nonetheless occur quantum mechanically because the framework constructed provides an extension of the reduced theory of general relativity which describes geometries of both the LC and the Kasner type and which remains meaningful even when the fundamental canonical variable $\tilde{A}$ on the reduced phase space vanishes, i.e., for degenerate metrics.

\section{QUANTUM GRAVITATIONAL FIELD OF A COSMIC STRING}

We have already commented that the LC metrics (1.1) with $p=\frac{1}{2}$ and $C>A B$ represent the exterior gravitational field of a straight cosmic string. With the aim of constructing a quantum framework for the analysis of the physical consequences that cosmic strings may imply, we now want to discuss in detail the quantization of this subfamily of static and cylindrical solutions.

The collection of metrics that we are going to study can be written in the form (1.2). We will first show that these metrics can be obtained from the LC solutions by imposing the constraints 


$$
\begin{gathered}
\chi_{1} \equiv p-\frac{1}{2}=0, \\
\chi_{2} \equiv P_{p}-A\left(\ln A-\ln D_{0}-2 p\right)=0,
\end{gathered}
$$

where $D_{0}$ is a fixed positive constant, $D_{0}>0$.

Recalling that the symplectic structure of the reduced phase space of the LC solutions is given by Eq. (2.9), it is easy to check that the above equations provide a pair of second-class constraints, with $\left\{\chi_{1}, \chi_{2}\right\}=1$. The imposition of (4.1), (4.2) leads to a reduced model whose only degrees of freedom are the variables $A$ and $\tilde{P}_{A}$, the latter defined in Eq. (2.7) [with $|\tilde{C}|$ equal to the parameter $C$ of Eqs. (1.1), (1.2)]. Making use of Eq. (2.9), it is also straightforward to see that $A$ commutes with $\chi_{1}$ and $\chi_{2}$. Therefore, after reduction of the system, $A$ and $\tilde{P}_{A}$ continue to be canonically conjugate to each other, because their Poisson and Dirac brackets coincide. Thus, the symplectic form on the physical phase space of the considered reduced model is

$$
\Gamma_{R}=d A \wedge d \tilde{P}_{A}
$$

On the other hand, employing the definition of $P_{p}$ in Eq. (2.3) and that $A>0$, one can prove that constraint (4.2) is equivalent to the condition

$$
B=\frac{A}{D_{0}}
$$

Then, provided that constraints (4.1), (4.2) hold, the fourdimensional metrics (1.1) become

$$
d s^{2}=C\left(-d t^{2}+d \rho^{2}\right)+\frac{A^{2}}{D_{0}} \rho^{2} d \phi^{2}+D_{0} d z^{2},
$$

with $C, A \in \mathrm{R}^{+}$and $D_{0}>0$ fixed. In addition, from Eqs. (2.7) and (4.4), the variable $\tilde{P}_{A}$ takes now the expression

$$
\tilde{P}_{A}=\frac{1}{2}\left(\ln A-\ln D_{0}-\ln C\right)
$$

Finally, to arrive at metrics (1.2), it suffices to perform the following rescaling in Eq. (4.5):

$$
\rho^{\prime}=\sqrt{C} \rho, \quad t^{\prime}=\sqrt{C} t, \quad z^{\prime}=\sqrt{D_{0}} z
$$

This rescaling leaves invariant the domains of definition of the radial, time, and axial coordinates, and transforms Eq. (4.5) into

$$
d s^{2}=-d t^{2}+d \rho^{2}+E^{2} \rho^{2} d \phi^{2}+d z^{2},
$$

where we have suppressed the primes in the new coordinates and

$$
E=\frac{A}{\sqrt{C D_{0}}}
$$

If $E \leqslant 1$, metric (4.8) describes the exterior conical geometry of a straight cosmic string, with associated deficit angle equal to [see Eq. (1.3)]

$$
\Delta=2 \pi(1-E) .
$$

Thus, for classical solutions which represent the gravitational field of a cosmic string, the parameters $A$ and $C$ must satisfy

$$
A, C \in \mathbb{R}^{+} \text {and } \sqrt{C D_{0}} \geqslant A,
$$

the last inequality guaranteeing that $E \leqslant 1$. Introducing then the new variables

$$
Q=A \ln E, \quad P_{Q}=\ln (-\ln E),
$$

where, from Eq. (4.9),

$$
\ln E=\ln A-\frac{1}{2} \ln D_{0}-\frac{1}{2} \ln C,
$$

a simple computation proves that restrictions (4.11) can be rewritten as

$$
Q \in \mathbb{R}^{-}, \quad P_{Q} \in \mathbb{R} .
$$

On the other hand, using Eq. (4.6), the symplectic form (4.3) can be expressed as

$$
\Gamma_{R}=d Q \wedge d P_{Q}
$$

The symplectic structure of the reduced model under consideration can therefore be identified with that of the cotangent bundle over $\mathbb{R}^{-}$. Hence, the variables $Q$ and

$$
L_{Q}=Q P_{Q}
$$

provide a complete set of elementary observables. We can represent these observables as the following operators acting on the vector space of complex functions over $\mathrm{R}^{-}$:

$$
\hat{Q} \Psi(Q)=Q \Psi(Q), \quad \hat{L}_{Q} \Psi(Q)=-i\left(Q \partial_{Q}+\frac{1}{2}\right) \Psi(Q) .
$$

The physical inner product is determined by demanding the Hermiticity of the operators $\hat{Q}$ and $\hat{L}_{Q}$, which represent real classical observables. This fixes the inner product to be

$$
\langle\Phi, \Psi\rangle=\int_{\mathbb{R}^{-}} d Q \Phi^{*}(Q) \Psi(Q),
$$

so that the Hilbert space of physical states is $L^{2}\left(\mathbb{R}^{-}, d Q\right)$.

To close this section, we will show how one can get predictions for the observable $E$ from the quantum theory constructed. Notice that, from the point of view of the classical solutions (4.8), $E$ is the only physically relevant observable of the conical geometries studied.

From the second of relations (4.12), it follows that we can represent the observable $\ln (-\ln E)$ by the quantum operator $\hat{P}_{Q}$. Since the action of $\hat{L}_{Q}=\widehat{Q P}_{Q}$ has been chosen in Eq. (4.17) as the symmetric product of $\hat{Q}$ and $-i \partial_{Q}$, it seems natural to define the operator $\hat{P}_{Q}$ by

$$
\hat{P}_{Q} \Psi(Q)=-i \partial_{Q} \Psi(Q) .
$$

We conclude in this way that, given any quantum state $\Psi$, the expectation value 


$$
\left\langle\Psi, \hat{P}_{Q} \Psi\right\rangle=-i \int_{\mathbb{R}^{-}} d Q \Psi^{*}(Q) \partial_{Q} \Psi(Q)
$$

is that corresponding to the observable

$$
\ln (-\ln E)=\ln \left[\ln \left(\frac{2 \pi}{2 \pi-\Delta}\right)\right]
$$

where we have used Eq. (4.10) to write $E$ in terms of the deficit angle of the conical geometry.

\section{CONCLUSIONS}

We have succeeded in quantizing the LC family of static and cylindrical solutions by applying the extended canonical program proposed by Ashtekar. In order to achieve this goal, we have first proved that the reduced phase spaces which correspond, respectively, to the LC metrics and to the Kasner model have the same symplectic structure. The distinction between these two types of spacetimes amounts to a different domain of definition for one of the phase space variables (namely, $P_{A}$ ). Taking this into account, the quantization of the LC solutions has been easily carried out by following a procedure parallel to that employed for the Kasner metrics in Ref. [20].

We have also constructed a quantum model which describes the LC and the Kasner solutions as a whole. This has been possible because the physical phase spaces of these two types of spacetimes possess the same symplectic structure and can be joined together to form a single phase space by including some degenerate metrics. The spatial topology of the model quantized is not uniquely fixed, for the topology of the surfaces of constant time is different in the LC and in the Kasner spacetimes, respectively. We have shown that, owing to this fact, some physical measurements can lead to a change in the spatial topology predicted by the constructed quantum theory.

Finally, we have quantized the subfamily of LC metrics which provide the exterior conical geometry of an infinite, straight cosmic string. These metrics can be obtained from the LC solutions by imposing a pair of second-class constraints and demanding that the deficit angle of the fourdimensional geometry be positive. The last requirement guarantees that the global geometry is conical. In this way, one reaches a reduced model whose physical phase space can be identified with the cotangent bundle over $\mathbb{R}^{-}$, and which can be quantized following standard methods. The corresponding quantum theory allows us to get predictions about the value of the deficit angle caused by the existence of a straight cosmic string.

\section{ACKNOWLEDGMENTS}

The author is grateful to P. F. González Díaz for helpful conversations. This work was supported by funds provided by DGICYT and the Spanish Ministry of Education and Science under Contract Adjunct to the Project No. PB91-0052.
[1] A. S. Kompaneets, Sov. Phys. JETP 7, 659 (1958).

[2] L. Marder, Proc. R. Soc. London A244, 524 (1958).

[3] K. S. Thorne, Phys. Rev. 138, B251 (1965).

[4] J. J. Stachel, J. Math. Phys. 7, 1321 (1966).

[5] E. Verdaguer, Phys. Rep. 229, 1 (1993), and references therein.

[6] V. A. Belinskiı̌ and V. E. Zakharov, Sov. Phys. JETP 48, 985 (1978).

[7] T. Levi-Cività, Rend. Accad. Lincei 28, 3 (1919); 28, 101 (1919)

[8] D. Kramer, H. Stephani, M. MacCallum, and E. Herlt, Exact Solutions of Einstein's Field Equations (Cambridge University Press, Cambridge, England, 1980).

[9] W. Israel, Phys. Rev. D 15, 935 (1977).

[10] A. Vilenkin, Phys. Rep. 121, 263 (1985); in Three Hundred Years of Gravitation, edited by S. W. Hawking and W. Israel (Cambridge University Press, Cambridge, England, 1987).

[11] J. R. Gott, Astrophys. J. 288, 422 (1985); W. A. Hiscock, Phys. Rev. D 31, 3288 (1985); B. Linet, Gen. Relativ. Gravit. 17, 1109 (1985).

[12] T. W. B. Kibble, J. Phys. A 9, 1387 (1976); Phys. Rep. 67, 183 (1980).

[13] A. Linde and D. Linde, Phys. Rev. D 50, 2456 (1994).

[14] Ya. B. Zel'dovich, Mon. Not. R. Astron. Soc. 192, 663 (1980); A. Vilenkin, Phys. Rev. Lett. 46, 1169, 1496(E) (1981); A. Vilenkin and Q. Shafi, ibid. 51, 1716 (1983).

[15] N. Kaiser and A. Stebbins, Nature (London) 310, 391 (1984);
R. H. Brandenberger and N. Turok, Phys. Rev. D 33, 2182 (1986); T. Vachaspati, Nucl. Phys. B277, 593 (1986); A. Vilenkin, Nature 322, 613 (1986).

[16] A. Vilenkin, Phys. Rev. D 23, 852 (1981); Astrophys. J. Lett. 282, L51 (1984); B. Paczynski, Nature 319, 567 (1986).

[17] P. A. M. Dirac, Lectures on Quantum Mechanics, Belfer Graduate School of Science Monograph Series No. 2 (Yeshiva University, New York, 1964).

[18] A. Ashtekar, in Lectures on Non-Perturbative Canonical Gravity, edited by L. Z. Fang and R. Ruffini (World Scientific, Singapore, 1991); in Gravitation and Quantizations, proceedings of the Les Houches Summer School of Theoretical Physics, Session 57, Les Houches, France, 1992, edited by B. Julia and J. Zinn-Justin (North-Holland, Amsterdam, 1995).

[19] A. Ashtekar and J. Pullin, Ann. Israel Phys. Soc. 9, 65 (1990); H. Kastrup and T. Thiemann, Nucl. Phys. B399, 211 (1993); H. Kodama, Phys. Rev. D 42, 2548 (1990); G. A. Mena Marugán, ibid. 50, 3923 (1994); Class. Quantum Grav. 11, 589 (1994); 11, 2205 (1994).

[20] N. Manojlović and G. A. Mena Marugán, Int. J. Mod. Phys. D (to be published).

[21] N. Manojlović and G. A. Mena Marugán, Phys. Rev. D 48, 3704 (1993); A. Ashtekar, R. S. Tate, and C. Uggla, Int. J. Mod. Phys. D 2, 15 (1993).

[22] A. Rendall, Class. Quantum Grav. 10, 2261 (1993). 